\title{
Synthesis and characterization of a new triptycene-based tripod
}

Jesús Sanmartín-Matalobos, ${ }^{\mathrm{a},}$, Ana M. García-Deibea , Matilde Fondo ${ }^{\mathrm{a}}$, J. Luis Pérez-Lustres ${ }^{\mathrm{b}}$, Sourav Bhowmick ${ }^{c}$ and Neeladri Das ${ }^{c}$

aDpto. de Química Inorgánica, Facultad de Química, Universidad de Santiago de Compostela, Avenida de las Ciencias s/ $n, 15782$ Santiago de Compostela, Spain.

Fax: +34 981 528073; Tel: +34 981 563100; E-mail: jesus.sanmartin@usc.es

${ }^{b}$ Centro Singular de Investigacion en Química Biológica y Materiales Moleculares (CIQUS),

Universidad de Santiago de Compostela, Jenaro de la Fuente s/n, 15782 Santiago de

Compostela, Spain

cDepartment of Chemistry, Indian Institute of Technology Patna, Patna 800 013, Bihar, India.

Abstract: Herein, we report an efficient and facile synthesis of a new triptycene-based tripodal ligand containing both imine functionality and phenolic pendant arms. This tripodal unit is a potential building block for constructing novel supramolecular architectures. The newly synthesized 2,6,14-triaminotriptycene derivative, which was characterized by FT-IR, UV-Vis absorption, mass and NMR spectroscopic techniques has interesting properties such as high solubility in common organic solvents and fluorescence emission in THF solution $(\lambda=550 \mathrm{~nm})$.

\section{Keywords}

Fluorescence emission / Triptycene derivatives / Schiff Bases / Tripods

\section{Introduction}

Triptycenes are a class of compounds derived from 9,10-dihydro-9,10$[1,2]$ benzenoanthracene. The use of triptycene-based tripodal linkers for constructing novel supramolecular architectures, such as 3D finite polyhedra/metallacages (e.g., trigonal prisms, trigonal bipyramids, double squares, adamantanoids, dodecahedra, etc) is in very early stage (Scheme 1). To the best of our knowledge, only a few triptycene-based nanoscalar cage compounds have been reported in the literature till date [1-5]. All these triptycene-based cages are examples of nanosized molecules where the building blocks are self-assembled and held together by means of covalent bonds. Chen [1] has described the synthesis of a nanosized molecular cage using 2,7,14-trihalotriptycenes as the starting synthon, while Mastalerz [2-4] has used 2,7,14-triaminotriptycene as the synthon. By using the latter one, Das [5] has reported the first example of a truly Pt(II) based trigonal bipyramidal supramolecular cage containing amide functionality (TBP in Scheme 1). 
The directional bonding approach dictates that the design of a polyhedral supramolecular structure necessitates one of the building blocks to possess at least three binding sites. Herein, we report an efficient and facile synthesis of a new triptycene-based tripodal ligand containing both imine functionality and phenolic pendant arms, and therefore, it can act as trisbidentate $\mathrm{N}, \mathrm{O}$-donor in neutral metal complexes of the type $\mathrm{M}_{3} \mathrm{~L}_{2}$.

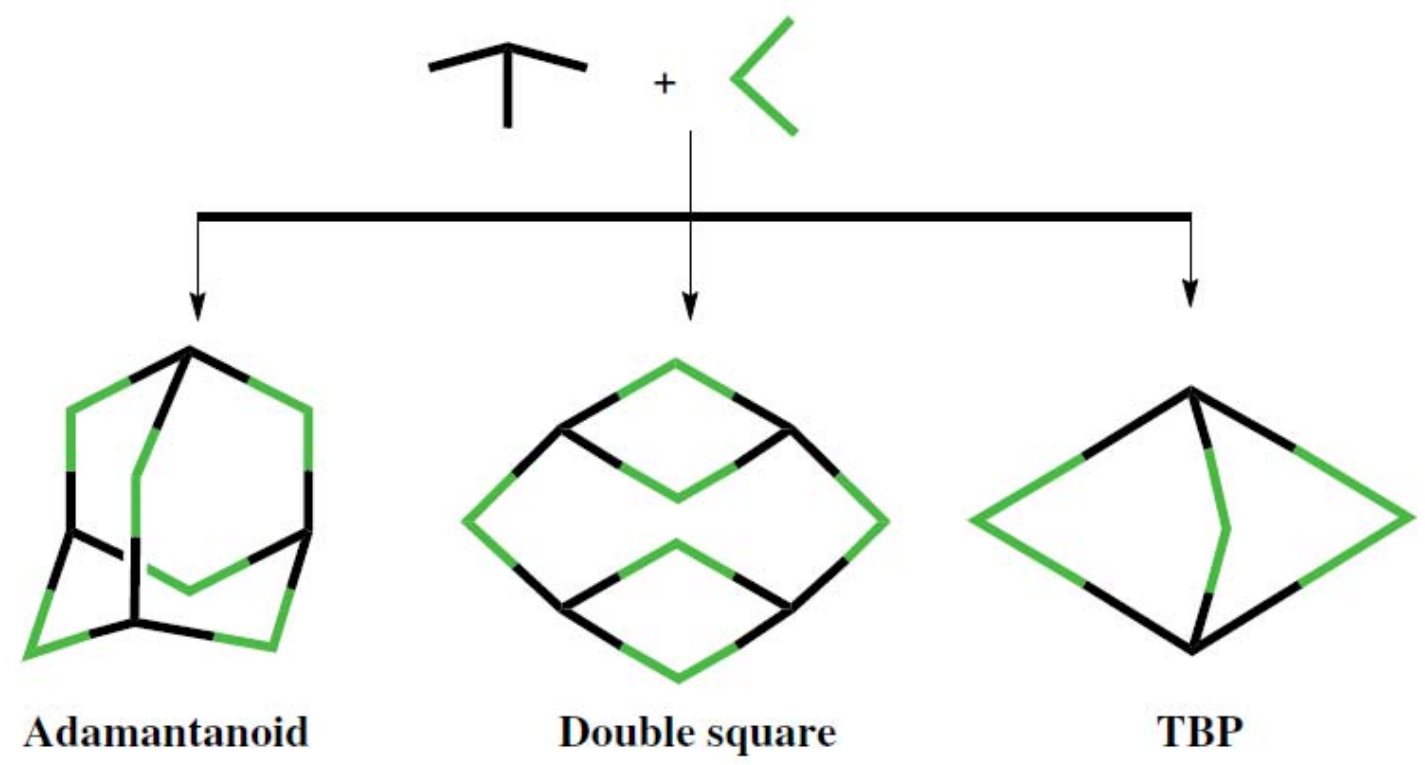

Scheme 1. Some of the metallacages that can be formed by reaction of a tripodal ligand (black) and a cis-protected metal acceptor with preference for square-planar coordination (green).

\section{Results and discussion}

Synthesis of the tripod. The triptycene-based tripodal ligand was synthesized by reacting 2-hydroxybenzaldehyde with 2,6,14-triaminotriptycene [6] in ethanol at room temperature (Scheme 2). The obtained tripod is very soluble in common organic solvents, such as chloroform, dichloromethane and toluene as well as other aromatic solvents, but it is rather insoluble in $\mathrm{MeOH}$ and $\mathrm{EtOH}$.

2,6,14-triaminotriptycene has been obtained according to literature [6]. First, 2,6,14trinitrotriptycene as the sole isolated product has been prepared by nitration of triptycene with concentrated $\mathrm{HNO}_{3}$ (Scheme 2). 2,6,14-trinitrotriptycene could be easily reduced by Raney $\mathrm{Ni}$ in the presence of hydrazine monohydrate to afford 2,6,14-triaminotriptycene. 


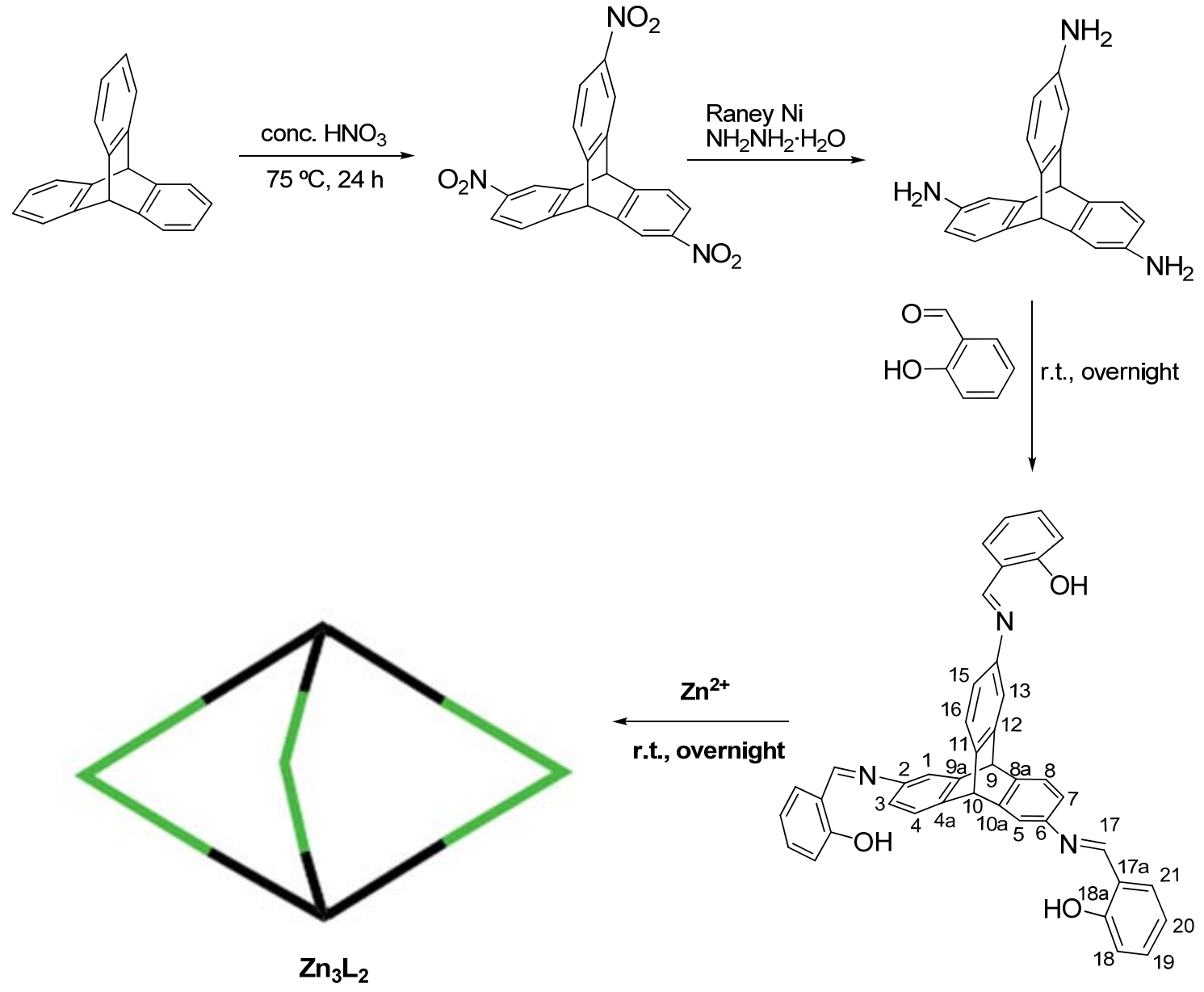

Scheme 2. Synthetic approach for obtaining the triptycene-based tripod for constructing TBP metallacages. The tripodal/trisbidentate N,O-donor ligand has been represented in black, while the four-coordinate metal acceptor has been represented in green.

Spectroscopic characterisation of the tripod. The structure of the triptycene-based tripod was elucidated on the basis of NMR, FT-IR, and mass spectroscopies.

${ }^{1} \mathrm{H}$ NMR spectrum of the triptycene-based tripod (Fig. 1) revealed the presence of three characteristic non-aromatic signals at about 13.0, 9.0 and $5.8 \mathrm{ppm}$ that were assigned to phenol $(3 \mathrm{H})$, imino $(3 \mathrm{H})$ and 9,10 protons of dihydroanthracene $(2 \mathrm{H})$, respectively. It must be noted that both phenol and imino proton signals of the arms in 2,6-positions appear as unique singlets at about 13.04 and $8.88 \mathrm{ppm}$, respectively, while the corresponding proton signals of the arm in 14-position are slightly shifted to lower field, which matches up almost exactly with the expected results. NMR assignment of each aromatic proton signal, which is shown in Fig. 1 (bottom), was made by a combination of COSY and NOESY experiments. 
The IR spectrum of the above triptycene-based tripod shows two strong absorption bands at about 1620 and $1610 \mathrm{~cm}^{-1}$ corresponding to the vibrations of the different imine groups of the molecule. Besides, a broad band corresponding to $\mathrm{OH}$ vibrations is observed at about $3430 \mathrm{~cm}^{-1}$. A MALDI-TOF mass spectrum shows the molecular peak ion at $611.7 \mathrm{~m} / \mathrm{z}$.

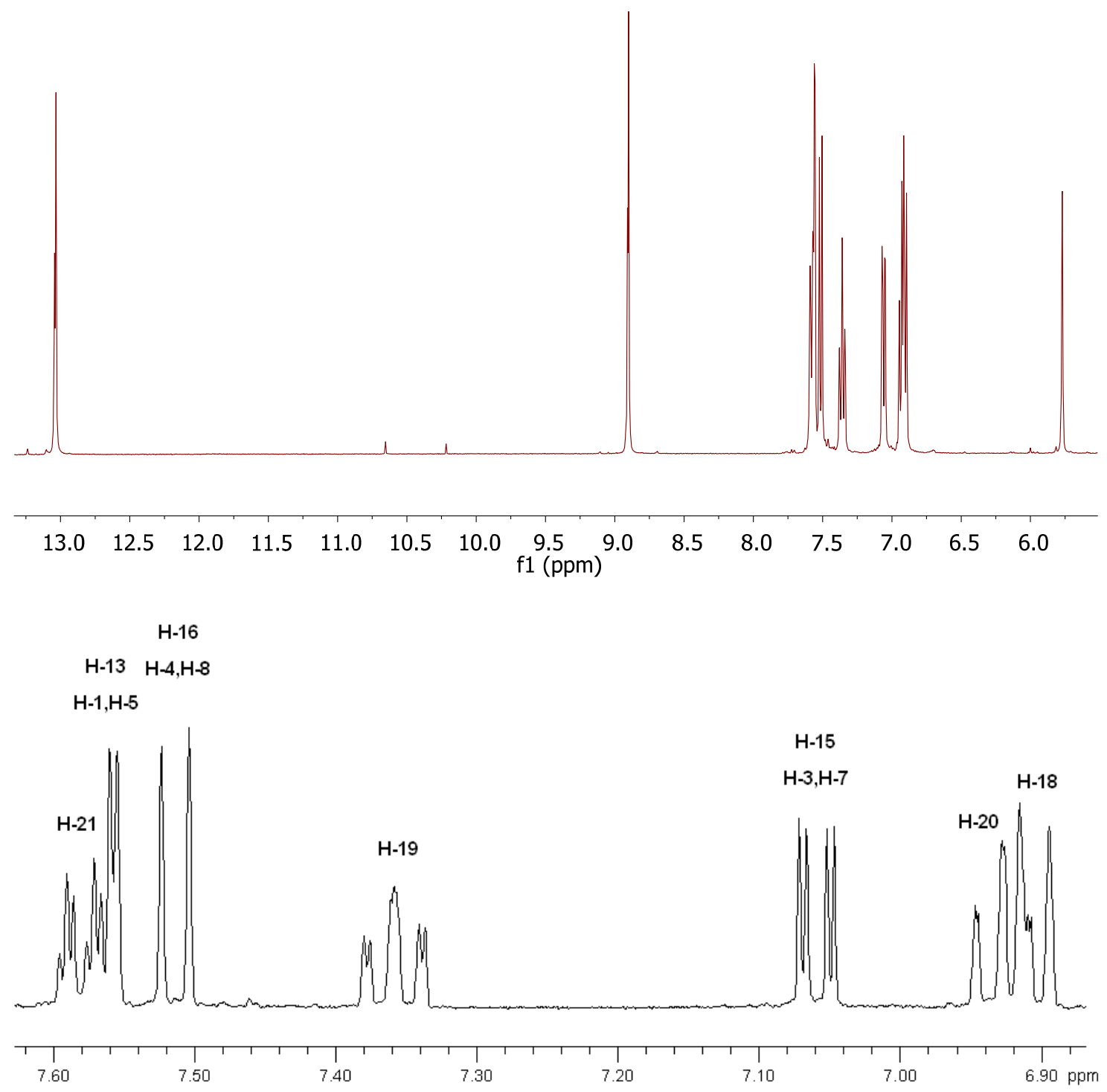

Fig. 1. View of the ${ }^{1} \mathrm{H}$ NMR spectrum of the triptycene-based tripod in dmso- $_{6}$ (top) with magnification of the aromatic region (bottom)

Both UV-Vis absorption spectrum and fluorescence emission spectrum of the triptycenebased tripod were measured at room temperature in dilute THF solutions (Fig. 2). The 
presence of an intense absorption band at about $350 \mathrm{~nm}$ is a characteristic spectral feature of the triptycene-based tripod. The emission spectrum displays a band at about $550 \mathrm{~nm}$, by excitation of the solved compound at $350 \mathrm{~nm}$.

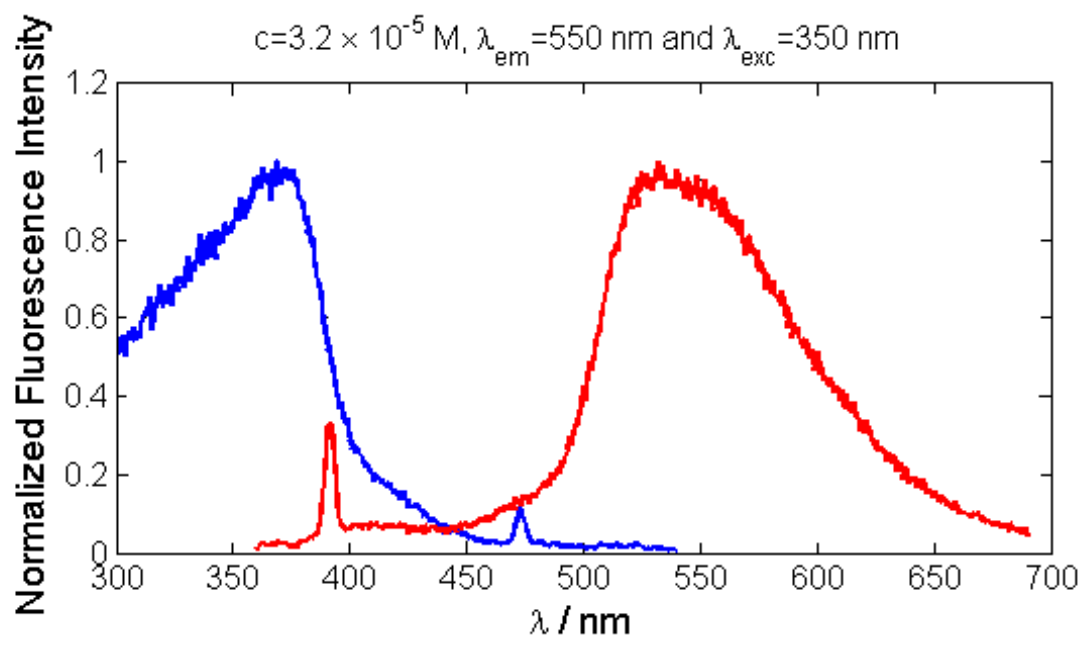

Fig. 2. UV-Vis absorption (left) and fluorescence emission (right) spectra of the triptycene-based tripod in THF

To illustrate that the prepared triptycene-based tripod is a potential building block for constructing novel supramolecular architectures, we reacted the tripodal unit with $\mathrm{Zn}(\mathrm{OAc})_{2} \cdot 2 \mathrm{H}_{2} \mathrm{O}$ in 2:3 stoichiometric ratio. This self-assembly reaction yielded the expected trigonal bipyramidal cage [5] as a sole isolated product ( $\mathrm{Zn}_{3} \mathrm{~L}_{2}$ in Scheme 2). The ${ }^{1} \mathrm{H}$ NMR spectrum (in dmso- $_{6}$ ) of this product showed the absence of phenol protons, which is indicative of trideprotonation of the ligand by coordination to $\mathrm{Zn}$ (II) metal centres. Besides, a typical high-field chemical shift (about $0.4 \mathrm{ppm}$ ) of the imino protons of the arms in 2,6- and 14-positions clearly shows the participation of the azomethine groups in the coordination. Mass spectrum of the compound supported the formation of the $\mathrm{Zn}_{3} \mathrm{~L}_{2}$ compound.

\section{Conclusions}

We report the synthesis of a supramolecular donor building block having a central triptycene unit and decorated with three phenolic pendant arms. The new molecule has been characterized by elemental analysis, FT-IR, NMR, UV-Vis and MALDI-TOF spectrometries. The triptycene-based tripodal ligand acting as trisbidentate N,O-donor drives self-assembly processes with naked $\mathrm{Zn}$ (II) ions that leads to a TBP metallacage. Interestingly, the triptycene-based tripod is soluble in common organic solvents and emits fluorescence in solution. 


\section{Experimental}

Syntheses of the triptycene-based tripod

A solution of 2,6,14-triaminotriptycene $(100 \quad \mathrm{mg}, \quad 0.334 \mathrm{mmol})$ and 2-hydroxybenzaldehyde $(0.11 \mathrm{~mL}, 1.003 \mathrm{mmol})$ in ethanol $(10 \mathrm{~mL})$ with $4-5$ drops of acetic acid was stirred at room temperature for overnight. Then, it was obtained a yellow solid, which was washed with methanol to get the desired product. Yield: above $90 \% .{ }^{1} \mathrm{H}$ NMR (400 MHz, dmso-d $\left.d_{6}, \mathrm{ppm}\right) 13.05$ (s, 2H, 1xOH), 13.04 (s, 2H, 2xOH), 8.89 (s, 1H, 1xH17), 8.88 (s, 2H, 2xH-17), 7.57 (d, 3H, 3xH-21), 7.55 (d, 3H, H-1/H-5, H-13), 7.52 (d, 3H, H-4/H-8, H-16), 7.35 (t, 3H, 3xH-19), 7.06 (d, 3H, H-3/H-7, H-15), 6.92 (t, 3H, 3xH-20), 6.90 (d, 3H, 3xH-18), 5.71 (s, 2H, H-9/H-10). IR (KBr, v/cm-1): $3430(\mathrm{OH}), 1625$ (C=N). MS (MALDI-TOF) $m / z(\%): 611.7$ (100) $\left[\mathrm{H}_{2} \mathrm{~L}+\mathrm{H}\right]^{+}$. Elemental analysis (Found): C, 80.9; H, 4.6; N, $6.6 \%$. Calcd. for $\mathrm{C}_{41} \mathrm{H}_{29} \mathrm{~N}_{3} \mathrm{O}_{3} \mathrm{C}, 80.5 ; \mathrm{H}, 4.7 ; \mathrm{N}, 6.9 \%$.

Syntheses of the tbp cage

Self-assembly of the triptycene tripod $\left(0.030 \mathrm{~g}, 4.910^{-5} \mathrm{~mol}\right.$ in $7 \mathrm{~mL}$ of DCM) with $\mathrm{Zn}(\mathrm{OAc})_{2} \cdot 2 \mathrm{H}_{2} \mathrm{O}\left(0.016 \mathrm{~g}, 7.410^{-5} \mathrm{~mol}\right.$ in $7 \mathrm{~mL}$ of $\left.\mathrm{MeCN}\right)$ in a 2:3 ratio was carried out at room temperature for overnight and the resulting solution was evaporated and washed with diethyl ether to obtain the cage $\mathrm{Zn}_{3} \mathrm{~L}_{2}$ as yellow coloured powdered solid. ${ }^{1} \mathrm{H}$ NMR (400 MHz, dmso- $\left.d_{6}, \mathrm{ppm}\right) 8.50$ (s, $1 \mathrm{H}, 2 \mathrm{xH}-17$ ), 8.44 (s, 2H, 1xH-17), 7.60-6.50 (aromatic protons) 5.65 (s, 1H, H-9/H-10), 5.55 (s, 1H, H-9/H-10). MS (MALDI-TOF+) m/z (\%): $1412.2\left[\mathrm{Zn}_{3} \mathrm{~L}_{2}+\mathrm{H}\right]^{+}$.

\section{References}

[1] Zhang, C.; Chen, C.-F. J. Org. Chem. 2007, 72, 9339;

[2] Schneider, M. W.; Oppel, I. M.; Ott, H.; Lechner, L. G.; Hauswald, H.-J. S.; Stoll, R.; Mastalerz, M. Chem. Eur. J. 2012, 18, 836

[3] Schneider, M. W.; Hauswald, H.-J. S.; Mastalerz, M. Chem. Commun. 2012, 48, 9861

[4] Schneider, M. W.; Oppel, I. M.; Mastalerz, M. Chem. Eur. J. 2012, 18, 4156.

[5] Chakraborty, S.; Mondal, S.; Li, Q.; Das N. Tetrahedron Letters, 2013, 54, 1681.

[6] Zhang, C.; Chen, C.-F. J. Org. Chem. 2006, 71, 6626. 